\title{
Hemodialysis Patient Outcomes: Provider Characteristics
}

\author{
Yelena Slinin ${ }^{a, b}$ Haifeng Guo ${ }^{c}$ Suying $\mathrm{Li}^{c}$ Jiannong Liu ${ }^{c}$ Kristine Ensrud ${ }^{a, b}$ \\ David T. Gilbertson ${ }^{c}$ Allan J. Collins ${ }^{b, c}$ Areef Ishani ${ }^{a-c}$ \\ a Veterans Administration Health Care System, ${ }^{b}$ Department of Medicine, University of Minnesota, and \\ ${ }^{c}$ Chronic Disease Research Group, Minneapolis Medical Research Foundation, Minneapolis, Minn., USA
}

\author{
Key Words \\ Hemodialysis - Patient outcomes · Provider characteristics . \\ Quality of care
}

\begin{abstract}
Background/Aims: Physician characteristics are associated with differential performance on quality measures and patient outcomes in several medical fields. We aimed to determine whether characteristics of physicians who provide care to dialysis patients were associated with patient outcomes. Methods: This cohort study used United States Renal Data System data for patients who initiated in-center hemodialysis between October 1, 2003, and September 30, 2006 ( $\mathrm{n}=$ $91,276)$. Patient characteristics were defined and physicians identified from Part B Medicare claims for outpatient dialysis services submitted during months 4-6 of hemodialysis. Physician characteristics were obtained from the American Medical Association Physician Master File. Associations of physician characteristics with 1-year patient mortality and first hospitalization were determined using Cox proportional hazards analysis; associations with quality of care (defined by influenza vaccination and waitlisting for kidney transplant) were determined using logistic regression. Results: Physician characteristics were not associated with patient mortality. After adjustment for patient and other provider characteristics, patients whose physicians had practiced longer or were in administrative, research, or teaching practices were more likely to be hospitalized; patients whose provid-
\end{abstract}

ers practiced in smaller metropolitan statistical areas (MSAs) were less likely. More years since training was associated with greater chance of waitlisting, and practicing in smaller MSAs with less chance. Graduation from a foreign medical school, practicing in smaller MSAs, and travelling farther from office to dialysis unit were associated with greater odds of influenza vaccination. Conclusions: Several characteristics of physicians seeing incident outpatient hemodialysis patients were associated with hospitalization and quality of care, but none with mortality.

(c) 2014 S. Karger AG, Basel

\section{Introduction}

Physician characteristics have been associated with differential performance on quality measures and patient outcomes in several medical fields $[1,2]$. Studies investigating the association between provider years of experience [2], board certification [3], practice size [3], and attendance of domestic medical schools [3] with quality of care, most frequently defined as adherence to clinical practice guidelines, have reported conflicting results. The few studies that have reported an association between provider characteristics and patient clinical outcomes such as hospitalizations and mortality have focused on mortality after acute myocardial infarction [4-7] or on surgical outcomes [8]. Larger nephrologist case load was associated with higher dialysis patient mortality in an ur-

\section{KARGER}

E-Mail karger@karger.com

www.karger.com/ajn
(C) 2014 S. Karger AG, Basel

0250-8095/14/0395-0367\$39.50/0
Yelena Slinin, MD, MS

VA Medical Center (111J)

One Veteran's Drive

Minneapolis, MN 55417 (USA)

E-Mail slini001@umn.edu 
ban cohort of prevalent dialysis patients [9]. Whether other characteristics of physicians who care for hemodialysis patients are associated with patient morbidity and mortality is unknown. In a cohort of incident hemodialysis patients, we aimed to determine if hemodialysis provider characteristics were associated with patient outcomes, including quality of care (defined by influenza vaccination and waitlisting for kidney transplant), morbidity or mortality.

\section{Methods}

The study cohort consisted of incident in-center hemodialysis patients who initiated in-center hemodialysis between October 1, 2003, and September 30, 2006, and had Medicare as primary payer, Part A and Part B, at day 91 after initiation; were aged 20 years or older and resided in the US; survived and continued in-center hemodialysis for the first 6 months of end-stage renal disease (ESRD), and were not hospitalized during at least 1 month of months 4-6 of ESRD. We excluded patients who had undergone previous kidney transplant. Patients whose physicians did not have an available valid Universal Provider Identification Number (UPIN) were also excluded.

Data were from the United States Renal Data System (USRDS), including all Medicare claims (Part A institutional, Part B physician/supplier), Medicare enrollment data, ESRD Medical Evidence Report (Centers for Medicare \& Medicaid, CMS, form CMS-2728) data, and ESRD Death Notification (form CMS-2746) data.

Because dialysis patients are eligible for Medicare coverage 3 months after dialysis initiation, we used a 3-month run-in period, consisting of months 4 through 6 , to identify primary hemodialysis providers and to define patient characteristics. Providers who billed in the most months for a given patient's outpatient dialysis care during the 3-month run-in period or cared for the patient during the last month of the run-in period (if providers for all months differed or if only 1 month was used to define the provider) were considered providers of record. We used calendar months to define the run-in period because provider bills for dialysis visits occur on the calendar cycle. The start date for patient follow-up was the first day after the run-in period. For analyses of waitlisting for kidney transplant and influenza immunization, patients were required to have survived for 1 year after the run-in period (through month 18 of ESRD).

We identified physicians providing care to hemodialysis patients using the UPIN from Part B Medicare claims for outpatient dialysis services submitted during the run-in period. We were able to identify only providers of comprehensive visits whose UPIN numbers appeared on the Medicare claims. We determined physician characteristics by merging our data with the American Medical Association Physician Master File by UPIN. We counted providers as nephrologists if they indicated nephrology as their primary or secondary subspecialty. Travel distance between provider office and dialysis facility was calculated based on the provider's billing zip code and the zip code of the respective dialysis facility. Number of patients per provider was calculated as the number of incident and prevalent hemodialysis pa- tients for whose care an individual provider billed Medicare during the year. Provider-patient visit frequency was calculated as the mean number of visits per month by a dialysis provider to each patient during the 3-month run-in period. Visit frequency information was obtained using Medicare Part B claims code G0319 as 1 visit, code G0318 as 2.5 visits, and code G0317 as 4 visits. For patients hospitalized for any portion of a month during the run-in period, that month's provider visit frequency was counted as missing.

Information on patient demographics, comorbid conditions, and laboratory values at dialysis initiation was obtained from the Medical Evidence Report. Comorbid conditions were defined based on the Medical Evidence Report and using International Classification of Diseases, Ninth Revision, Clinical Modification codes from Medicare claims incurred during days 0-180 of ESRD [10]. The comorbidity index for dialysis patients was calculated based on 11 comorbid conditions [11]. A socioeconomic status (SES) score was determined for each patient based on home zip code using 2000 census data [12]. We also used dual eligibility for Medicaid and Medicare as a surrogate for low SES. Rural and urban definitions were adopted from the US Census Bureau based on zip code [13]. Facilities were identified from Medicare claims for dialysis and characteristics were obtained from the USRDS.

The primary outcome measure was mortality. Death information was obtained from the USRDS, which receives and reconciles death dates from several sources, including the CMS Enrollment Database, form CMS-2746 (ESRD Death Notification), the ESRD Network database, and the Social Security Death Master File. Secondary outcome measures were time to first hospitalization, waitlisting for kidney transplant, and receiving an influenza vaccination during the year of follow-up. Hospitalization information was obtained from Part A Medicare inpatient claims. Patients who were included in the influenza vaccination analysis were required to survive for at least 1 year following cohort entry to ensure one influenza season. Influenza vaccinations were identified by CPT codes 90724, 90657, 90658, 90659, and 90660, and HCPCS code G0008 during the year following the cohort entry. We chose these indicators of care quality based on their availability in our research database. Our dataset included no information about dialysis dose or management of bone mineral metabolism.

\section{Statistical Analyses}

We conducted unadjusted analyses for time to death, time to first hospitalization, and time to waitlisting for kidney transplant using the Kaplan-Meier method. A Cox proportional hazard regression model was used to assess the association between provider characteristics and time to death and time to first hospitalization. The robust standard error method was used to account for clustering of patients by nephrologist. For mortality analyses, patients were followed until death, change of renal replacement modality, recovery of kidney function, or 1 year. For the hospitalization outcome, patients who changed insurance type were additionally censored. Multivariate logistic regression was used to evaluate the association between provider characteristics and waitlisting for kidney transplant and influenza vaccination. The generalized estimating equations (GEE) method was used to account for clustering of patients by nephrologist. Several variables, such as years since end of physician training, miles from physician's office to patient's dialysis unit, and number of hemodialysis patients per 
physician, were expressed as tertiles for the primary analyses and as continuous variables for sensitivity analyses. We performed regression spline analyses to evaluate an association between years since end of physician training, miles from physician's office to patient's dialysis unit, and number of hemodialysis patients per physician and mortality and hospitalizations. All analyses were performed using 9.1 SAS software.

\section{Results}

Of the 91,276 patients included in the cohort, $63.2 \%$ were aged 65 years or older, $28.3 \% 45-64$ years, and $8.5 \%$ 20-44 years; $47.0 \%$ were women; $65.6 \%$ were white, 29.7\% African American, and 11.5\% Hispanic; $48.7 \%$ had ESRD secondary to diabetes; $38.2 \%$ had a comorbidity score of 4 or less and $36.9 \%$ of 8 or more, and $70.5 \%$ lived in urban areas (table 1). Most patients were cared for by male providers ( $84.5 \%) ; 29.1 \%$ of patients were cared for by providers who had been in practice for 8 years or less and $33.8 \%$ by providers who had been in practice for 22 years or longer; $44.1 \%$ of patients were cared for by providers who had graduated from foreign (non-domestic) medical schools (table 2). Nephrologists provided outpatient dialysis care for $93.1 \%$ of patients; providers with office-based practices cared for $88.2 \%$, and providers in administrative, teaching, or research positions for $2.4 \%$. Most patients $(52.3 \%)$ received care from providers who practiced in large metropolitan areas of 1 million or more population. Providers for one third of patients drove more than 9.8 miles from their primary office to the dialysis unit; providers for $39.1 \%$ of patients cared for more than 100 dialysis patients over the course of the year.

In unadjusted analyses, risk of death at 1 year was lower for patients of female providers than for patients of male providers (hazard ratio, HR, 0.94, 95\% confidence interval, CI, 0.90-0.98); risk of death was higher for patients of providers who had been in practice longer (HR $1.06,95 \%$ CI 1.02-1.10, for providers in practice for 9-21 years, and 1.01, 0.98-1.05, for providers in practice $\geq 22$ years, compared with providers in practice for $\leq 8$ years). Risk of death was lower for patients of providers with full-time hospital practice (HR 0.92, 95\% CI 0.86-0.99, compared with patients of office-based providers). Risk of death was higher for patients whose providers practiced in smaller communities than for patients whose providers practiced in larger metropolitan areas (HR $1.06,95 \%$ CI 1.01-1.11, for metropolitan statistical area, MSA, population 100,000-249,999, and 1.08, 1.03-1.13, for MSA population $<100,000$ compared with MSA pop-
Table 1. Baseline characteristics of incident in-center hemodialysis patients (October 1, 2003, to September 30, 2006)

\begin{tabular}{|c|c|}
\hline Total patients & 91,276 \\
\hline \multicolumn{2}{|l|}{ Physician visits per month } \\
\hline$<4$ & $35,891(39.3)$ \\
\hline 4 & $55,385(60.7)$ \\
\hline \multicolumn{2}{|l|}{ Age } \\
\hline $20-44$ years & $7,795(8.5)$ \\
\hline $45-64$ years & $25,786(28.3)$ \\
\hline$\geq 65$ years & $57,695(63.2)$ \\
\hline Females & $42,876(47.0)$ \\
\hline \multicolumn{2}{|l|}{ Race } \\
\hline White & $59,831(65.6)$ \\
\hline Black & $27,100(29.7)$ \\
\hline Other & $4,345(4.8)$ \\
\hline Hispanic & $10,511(11.5)$ \\
\hline \multicolumn{2}{|l|}{ Primary cause of ESRD } \\
\hline Diabetes & $44,465(48.7)$ \\
\hline Hypertension & $27,890(30.6)$ \\
\hline Glomerulonephritis & $5,393(5.9)$ \\
\hline Other/missing/unknown & $13,528(14.8)$ \\
\hline \multicolumn{2}{|l|}{ Comorbidity score } \\
\hline$\leq 4$ & $34,843(38.2)$ \\
\hline$>4$ to $<8$ & $22,740(24.9)$ \\
\hline$\geq 8$ & $33,693(36.9)$ \\
\hline SES/principal component less than median & $45,213(49.5)$ \\
\hline Eligible for Medicaid & $27,641(30.3)$ \\
\hline Urban residence & $64,385(70.5)$ \\
\hline \multicolumn{2}{|l|}{ Dialysis sessions during run-in } \\
\hline$<39$ & $28,900(31.7)$ \\
\hline$\geq 39$ & $62,376(68.3)$ \\
\hline \multicolumn{2}{|l|}{ Hospital days during run-in } \\
\hline 0 & $62,241(68.2)$ \\
\hline$>0$ to $<8$ & $16,443(18.0)$ \\
\hline$\geq 8$ & $12,592(13.8)$ \\
\hline \multicolumn{2}{|l|}{$\mathrm{eGFR}, \mathrm{ml} / \mathrm{min} / 1.73 \mathrm{~m}^{2}$} \\
\hline$<10$ & $49,420(54.1)$ \\
\hline$\geq 10$ & $41,856(45.9)$ \\
\hline \multicolumn{2}{|l|}{ Hemoglobin, g/dl } \\
\hline$<11$ & $64,551(70.7)$ \\
\hline$\geq 11$ & $26,725(29.3)$ \\
\hline \multicolumn{2}{|l|}{ Serum albumin, $\mathrm{g} / \mathrm{dl}$} \\
\hline$<3.4$ & $33,923(37.2)$ \\
\hline$\geq 3.4$ & $57,353(62.8)$ \\
\hline \multicolumn{2}{|l|}{ Body mass index } \\
\hline$<18.5$ & $3,462(3.8)$ \\
\hline 18.5 to $\leq 25$ & $30,515(33.4)$ \\
\hline$>25$ & $57,299(62.8)$ \\
\hline
\end{tabular}

eGFR $=$ Estimated glomerular filtration rate. Figures in parentheses indicate percentages.

ulation $\geq 1,000,000$ ). After adjustment for patient and other provider characteristics, none of the provider characteristics was associated with mortality risk (table 2). Expressing years since end of physician training, miles from physician's office to patient's dialysis unit, and number 
Table 2. Provider characteristics and patient mortality

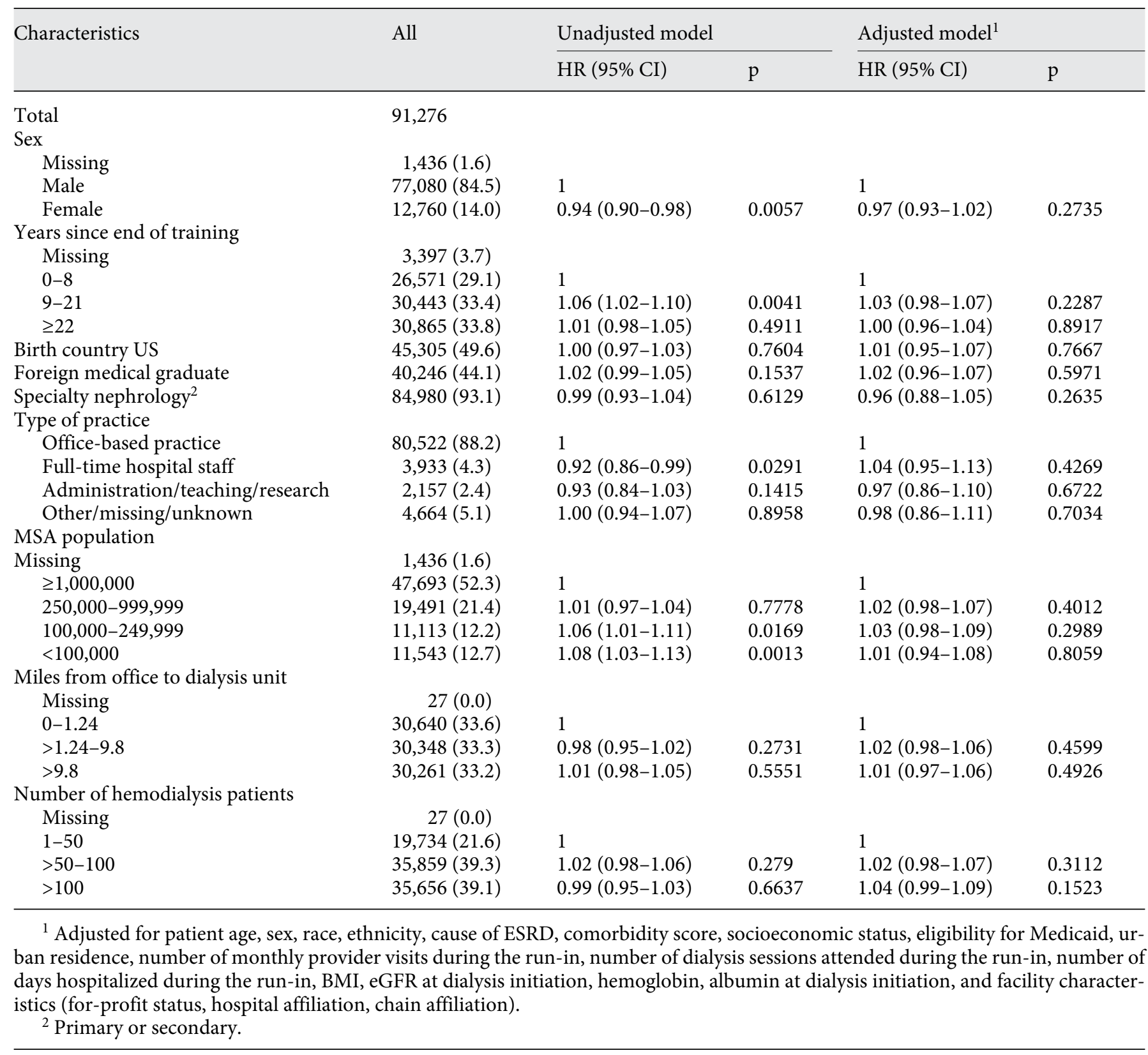

of hemodialysis patients per physician as continuous variables did not change the results, except that there appears to be a U-shaped association between years since end of training and patient survival $(p=0.017)$. The risk of mortality increased with increased years from end of training before year 10, was stable between years 10 and 25 , and then decreased sharply after year 25 . This result matches the result of categorical years since the end of training, the variable presented in table 2.
In unadjusted analyses, patients whose providers had been in practice longer were more likely to be hospitalized at 1 year (HR 1.03, 95\% CI 1.01-1.05, for practice of 9-21 years, and 1.00, $0.98-1.02$, for practice of $\geq 22$ years compared with practice of $0-8$ years); patients of providers in administrative, teaching, and research practices were more likely to be hospitalized than patients of providers in office-based practice (HR 1.06, 95\% CI 1.001.11). A similar U-shaped association between number of 
Table 3. Provider characteristics and hospitalization

\begin{tabular}{|c|c|c|c|c|}
\hline \multirow[t]{2}{*}{ Characteristics } & \multicolumn{2}{|l|}{ Unadjusted model } & \multicolumn{2}{|l|}{ Adjusted model ${ }^{1}$} \\
\hline & HR (95\% CI) & $\mathrm{p}$ & HR (95\% CI) & $\mathrm{p}$ \\
\hline \multicolumn{5}{|l|}{ Sex } \\
\hline Male & 1 & & 1 & \\
\hline Female & $0.99(0.96-1.01)$ & 0.2004 & $0.99(0.96-1.02)$ & 0.3378 \\
\hline \multicolumn{5}{|l|}{ Years since end of training } \\
\hline $0-8$ & 1 & & 1 & \\
\hline $9-21$ & $1.03(1.01-1.05)$ & 0.0024 & $1.03(1.00-1.05)$ & 0.0211 \\
\hline$\geq 22$ & $1.00(0.98-1.02)$ & 0.8485 & $1.00(0.97-1.02)$ & 0.7045 \\
\hline Birth country US & $0.95(0.94-0.97)$ & $<0.0001$ & $0.99(0.96-1.02)$ & 0.4555 \\
\hline Foreign medical graduate & $1.06(1.04-1.07)$ & $<0.0001$ & $1.02(0.99-1.06)$ & 0.2108 \\
\hline Specialty nephrology ${ }^{2}$ & $0.98(0.95-1.01)$ & 0.2652 & $0.99(0.95-1.03)$ & 0.5466 \\
\hline \multicolumn{5}{|l|}{ Type of practice } \\
\hline Office-based practice & 1 & & 1 & \\
\hline Full-time hospital staff & $0.99(0.95-1.03)$ & 0.6022 & $1.04(0.99-1.08)$ & 0.1493 \\
\hline Administration/teaching/research & $1.06(1.00-1.11)$ & 0.0367 & $1.08(1.00-1.16)$ & 0.0571 \\
\hline Other/missing/unknown & $1.03(1.00-1.07)$ & 0.0825 & $1.05(0.97-1.13)$ & 0.2217 \\
\hline \multicolumn{5}{|l|}{ MSA population } \\
\hline$\geq 1,000,000$ & 1 & & 1 & \\
\hline $250,000-999,999$ & $0.94(0.92-0.96)$ & $<0.0001$ & $0.95(0.92-0.97)$ & $<0.0001$ \\
\hline $100,000-249,999$ & $0.97(0.95-1.00)$ & 0.0265 & $0.96(0.93-1.00)$ & 0.0226 \\
\hline$<100,000$ & $0.97(0.95-1.00)$ & 0.0202 & $0.98(0.94-1.02)$ & 0.2359 \\
\hline \multicolumn{5}{|l|}{ Miles from office to dialysis unit } \\
\hline $0-1.24$ & 1 & & 1 & \\
\hline$>1.24-9.8$ & $1.01(0.99-1.03)$ & 0.5374 & $0.99(0.97-1.01)$ & 0.4276 \\
\hline$>9.8$ & $0.97(0.95-0.99)$ & 0.0068 & $0.98(0.96-1.01)$ & 0.134 \\
\hline \multicolumn{5}{|l|}{ Number of hemodialysis patients } \\
\hline $1-50$ & 1 & & 1 & \\
\hline$>50-100$ & $0.98(0.96-1.01)$ & 0.125 & $0.99(0.96-1.01)$ & 0.2998 \\
\hline$>100$ & $0.97(0.95-0.99)$ & 0.0025 & $0.99(0.96-1.02)$ & 0.4652 \\
\hline
\end{tabular}

\footnotetext{
${ }^{1}$ Adjusted for patient age, sex, race, ethnicity, cause of ESRD, comorbidity score, socioeconomic status, eligibility for Medicaid, urban residence, number of monthly provider visits during the run-in, number of dialysis sessions attended during the run-in, number of days hospitalized during the run-in, BMI, eGFR at dialysis initiation, hemoglobin, albumin at dialysis initiation, and facility characteristics (for-profit status, hospital affiliation, chain affiliation).

${ }^{2}$ Primary or secondary.
}

years since end of training as a continuous variable and patient hospitalization was found $(\mathrm{p}=0.0001)$. Patients whose providers were born in the US were less likely to be hospitalized (HR 0.95, 95\% CI 0.94-0.97), while patients whose providers were foreign medical graduates were more likely to be hospitalized (HR 1.06, 95\% CI 1.04-1.07). Risk of hospitalization was slightly but significantly lower for patients of providers who practiced in smaller communities, who travelled farthest from office to dialysis unit, and who cared for the most dialysis patients (table 3). After adjustment for patient and other provider characteristics, longer practice duration (HR 1.03 , 95\% CI 1.00-1.05, for 9-21 years in practice com- pared with $0-8$ years), and administrative, research, or teaching practice $(1.08,1.00-1.16$, compared with officebased practice) were associated with greater risk of hospitalization, but both findings were of borderline statistical significance. Practice in smaller MSAs was associated with lower risk of hospitalization (HR 0.95, 95\% CI 0.920.97 , for population $250,000-999,999$, and $0.96,0.93-$ 1.00 , for population 100,000-249,999 compared with population $\geq 1,000,000$; table 3 ).

Provider characteristics were associated with several quality of care indicators. After adjustment for multiple patient and provider characteristics, more years in practice was independently associated with higher chance of 
Table 4. Provider characteristics and quality of care indicators ${ }^{1}$

\begin{tabular}{|c|c|c|c|c|}
\hline & \multicolumn{2}{|c|}{$\begin{array}{l}\text { Cox regression, waitlisting for } \\
\text { kidney transplant }\end{array}$} & \multicolumn{2}{|c|}{$\begin{array}{l}\text { Logistic regression, } \\
\text { influenza vaccination }\end{array}$} \\
\hline & OR $(95 \% \mathrm{CI})$ & $\mathrm{p}$ & OR $(95 \% \mathrm{CI})$ & $\mathrm{p}$ \\
\hline Total & 72,734 & & 72,734 & \\
\hline \multicolumn{5}{|l|}{ Sex } \\
\hline Male & 1 & & 1 & \\
\hline Female & $1.02(0.93-1.13)$ & 0.6376 & $1.01(0.95-1.07)$ & 0.773 \\
\hline \multicolumn{5}{|l|}{ Years since end of training } \\
\hline $0-8$ & 1 & & 1 & \\
\hline $9-21$ & $1.04(0.96-1.13)$ & 0.302 & $0.97(0.92-1.02)$ & 0.3047 \\
\hline$\geq 22$ & $1.15(1.05-1.25)$ & 0.0014 & $0.96(0.91-1.02)$ & 0.1913 \\
\hline Birth country US & $1.03(0.93-1.15)$ & 0.5676 & $1.04(0.97-1.12)$ & 0.2202 \\
\hline Foreign medical graduate & $0.94(0.84-1.05)$ & 0.2821 & $0.94(0.87-1.00)$ & 0.0539 \\
\hline Specialty nephrology ${ }^{2}$ & $0.96(0.3-1.11)$ & 0.5544 & $0.97(0.89-1.06)$ & 0.4816 \\
\hline \multicolumn{5}{|l|}{ Type of practice } \\
\hline Office-based practice & 1 & & 1 & \\
\hline Full-time hospital staff & $1.04(0.91-1.19)$ & 0.5614 & $0.90(0.82-1.00)$ & 0.0531 \\
\hline Administration/teaching/research & $0.99(0.82-1.20)$ & 0.9449 & $0.93(0.81-1.06)$ & 0.2721 \\
\hline Other/missing/unknown & $1.06(0.87-1.28)$ & 0.5688 & $0.98(0.86-1.12)$ & 0.7462 \\
\hline \multicolumn{5}{|l|}{ MSA population } \\
\hline$\geq 1,000,000$ & 1 & & 1 & \\
\hline $250,000-999,999$ & $0.94(0.86-1.03)$ & 0.203 & $1.06(1.00-1.12)$ & 0.06037 \\
\hline $100,000-249,999$ & $0.89(0.79-0.99)$ & 0.0351 & $1.29(1.20-1.38)$ & $<0.0001$ \\
\hline$<100,000$ & $0.84(0.74-0.95)$ & 0.0041 & $1.05(0.97-1.14)$ & 0.2287 \\
\hline \multicolumn{5}{|l|}{ Miles from office to dialysis unit } \\
\hline $0-1.24$ & 1 & & 1 & \\
\hline$>1.24-9.8$ & $0.94(0.88-1.02)$ & 0.1337 & $1.03(0.98-1.08)$ & 0.3141 \\
\hline$>9.8$ & $0.95(0.88-1.02)$ & 0.1643 & $1.06(1.01-1.11)$ & 0.0258 \\
\hline \multicolumn{5}{|l|}{ Number of hemodialysis patients } \\
\hline $1-50$ & 1 & & 1 & \\
\hline$>50-100$ & $0.93(0.86-1.00)$ & 0.0605 & $1.04(0.98-1.09)$ & 0.1682 \\
\hline$>100$ & $0.84(0.78-0.92)$ & $<0.0001$ & $1.04(0.99-1.11)$ & 0.1289 \\
\hline
\end{tabular}

${ }^{1}$ Adjusted for patient age, sex, race, ethnicity, cause of ESRD, comorbidity score, socioeconomic status, eligibility for Medicaid, urban residence, number of monthly provider visits during the run-in, number of dialysis sessions attended during the run-in, number of days hospitalized during the run-in, BMI, eGFR at dialysis initiation, hemoglobin, albumin at dialysis initiation, and facility characteristics (for-profit status, hospital affiliation, chain affiliation).

${ }^{2}$ Primary or secondary.

waitlisting for kidney transplant (adjusted odds ratio, AOR, 1.15 , 95\% CI 1.05-1.25, for $\geq 22$ years in practice compared with 0-8 years), and practice in smaller MSAs was associated with lower chance (AOR 0.89, 95\% CI $0.79-0.99$, for population $100,000-249,999$, and 0.84 , $0.74-0.95$, for population $<100,000$ compared with population $\geq 1,000,000)$. Following provider characteristics were associated with greater odds of their patient influenza immunization: practice in smaller MSAs (AOR 1.29, 95\% CI 1.20-1.38, for population 100,000-249,999 com- pared with population $\geq 1,000,000$ ), and longer travel distance from office to dialysis unit (AOR 1.06, 95\% CI 1.011.11 for $>9.8$ miles compared with 0-1.24 miles; table 4 ).

\section{Discussion}

In this large study of a national cohort of incident dialysis patients with Medicare as primary payer, we found that few provider characteristics were independently as- 
sociated with patient outcomes. Few studies have assessed the association between characteristics of physicians who provide outpatient dialysis care and clinical outcomes of their patients. Many of the physician factors we assessed are not modifiable; however, the goal of this study was to identify whether disparities in patient care exist, so such disparities could be targeted by future policies and interventions.

We found no independent association between provider characteristics and patient mortality. Several studies that examined the association between provider characteristics and patient mortality focused on mortality after acute myocardial infarction [4-7] or surgical outcomes [8]. One study found no difference in the riskadjusted 30-day and 1-year mortality rates after acute myocardial infarction between patients of international and Canadian medical graduates [4]. Several studies that examined the association between provider characteristics and 1-year patient mortality after acute myocardial infarction found lower mortality in patients of providers who were cardiologists [5-7], who cared for more acute myocardial infarction patients [5], whose graduation from medical school was more recent [5], and who were board certified [5]. In one of these studies [6], the association between provider specialty and lower mortality was attenuated by adjustment for patient characteristics, including functional limitations and disease severity at presentation. Studies that assessed surgical outcomes found an association between increased mortality and more years since the surgeon was licensed $[8,14]$ and lower surgeon volume $[8,14,15]$. The only study that evaluated the association between provider characteristics and patient mortality in medical elderly patients without preceding acute events found that providers with patient mortality in the higher quartiles were more likely to be male, to have larger practices, to see more patients per day, to prescribe more medications, and to bill more per year, but the study did not adjust the association for patient characteristics [16].

We did not find an association between nephrologists' case load and patient mortality. This finding differs from findings of Harley et al. [9], who studied prevalent patients undergoing dialysis in an urban Southern California area and reported higher mortality among those cared for by nephrologists with larger case loads. This difference likely reflects differences in patient populations and geographic distribution. The Harley et al. [9] study included only 41 nephrologists who practice in the greater Los Angeles area, and our study included all nephrologists who provided dialysis care to Medi- care patients in the United States in 2006. The association between provider case load and patient outcomes might differ by geographic region. In addition, our study only addresses physicians' Medicare patient load, and this could have led to exposure misclassification and contributed to negative results. Similarly to Harley et al. [9], we found that patients of providers with larger case loads were less likely to be waitlisted for kidney transplant. Possibly, providers with smaller case loads practice in academic facilities associated with transplant centers and are more likely to refer their patients for transplant. Although patients of providers with larger case loads were less likely to be registered on the transplant waiting list, their mortality rates did not differ from rates of patients with smaller case loads. Because transplant improves patient outcomes, it is possible that we would have found differences in mortality with longer duration of follow-up. Other possible reasons for lack of association between provider characteristics and patient mortality in our study include protocolbased management of dialysis patients that is largely independent of physicians, and small variability of care patterns due to widely implemented clinical practice guidelines. In addition, we performed extensive adjustment for patient and facility characteristics. Some of the previously seen associations can possibly be attributed to different case mix among physicians with certain characteristics.

More years since a provider completed training was associated with a small increase in the risk of hospitalizations of borderline statistical significance, while patients of providers who have been in practice for more than 22 years were more likely to be waitlisted for kidney transplant. Findings of studies that evaluated the association between physician age or number of years in practice and patient outcomes and quality of care have been inconsistent; some studies suggested lower adherence to screening guidelines among older physicians [2, 17-19], while other studies suggested no difference [1] or improved adherence among physicians in practice longer $[2,20]$. One can speculate that earlier studies reflect time before required recertification by the American Board of Internal Medicine, which applies to providers who received initial board certification after 1990, and that providers who recertify provide up-to-date care. Since more years since training was not associated with greater mortality in our study, it is possible that physicians who have been in practice longer are more likely to hospitalize their patients because of different practice style. Although guidelines for hospital admissions exist for some diseases (pneumo- 
nia), the decision to admit a patient to the hospital remains subjective.

Administrative, research, or teaching practice was also associated with a small increase in risk of patient hospitalization in our study. To the best of our knowledge, ours is the only study to evaluate the association between type of practice and patient outcomes. This finding may possibly reflect practice by trainees rather than providers themselves. Alternatively, the outpatient infrastructure for treating infections or access malfunction may be less than ideal in these practices. Again, more uniform guidelines for patient hospitalization and improved infrastructure to manage dialysis access problems might narrow the difference.

Risk of hospitalization is lower for patients of providers who practice in intermediate-sized MSAs (population 250,000-999,999 and 100,000-249,999) than for patients of providers who practice in large MSAs (population $\geq 1,000,000$ ). In addition, patients of providers who practice in intermediate-sized MSAs were more likely to receive influenza immunizations. Because we could not identify influenza vaccinations received in the hospital, it is possible that we were more likely to identify immunizations in patients with fewer hospitalizations. Lower rates of hospitalization in intermediate and small MSAs without compromised patient mortality suggest better triaging practices and ability to avoid preventable hospitalizations compared with large metropolitan areas. This phenomenon should be further investigated and leveraged in areas with high avoidable hospitalization rates. Alternatively, patients in small MSAs may be less likely to be waitlisted for transplant and possibly less likely to undergo transplant, therefore enriching the population with healthier patients who experienced fewer hospitalizations.

Our finding that patients of providers who practice in small MSAs were less likely to be waitlisted for kidney transplant differs from prior findings [21, 22], indicating that rates of kidney transplant and waitlisting are higher for patients who reside in small metropolitan and rural areas. The reason for this discrepancy is unclear but possibly related to the selected nature of the cohort. Our finding might be attributed to different quality of care in metropolitan areas of different sizes, with different patient populations or different availability of resources such as emergency departments, hospital beds, hospital coverage, distance to the nearest transplant center, and outpatient infrastructure for treating complications. Our results underscore the need to equalize patient access to important services such as kidney transplant. Educational interven- tions are needed that target providers who practice in areas removed from transplant centers and reinforce provider knowledge about patient outcomes after transplant, and help providers and their patients navigate the complex world of transplant referral and posttransplant care.

Odds of receiving an influenza vaccination were similar for patients of providers who graduated from nonUS medical schools. Foreign medical graduates practicing in the primary care fields had lower proportions of Medicare beneficiaries receiving diabetic eye examinations, mammograms, colon cancer screenings, and influenza and pneumococcal vaccinations than did US and Canadian medical graduates [3], and their performance was lower on quality measures of preventative and gender-specific care, but not acute care [1]. A Canadian study of postmyocardial infarction patient outcomes found that use of secondary prevention medications, cardiac procedures, and mortality of acute myocardial infarction patients did not differ by admitting physician country of medical education [4]. Of interest, while international medical graduates make up about $25 \%$ of the US physician work force, $44 \%$ of incident dialysis patients in our study were followed by international medical graduates. International graduates are overrepresented among nephrologists, and their patient outcomes are equivalent to those of US medical graduates.

This study evaluated the association between characteristics of outpatient dialysis providers, mostly physicians, and their patient outcomes. Our study has several strengths. It is highly generalizable to incident Medicare hemodialysis patients and nephrologists in the US. It is large and well powered to detect small differences. The analyses were adjusted for multiple patient, provider, and dialysis facility characteristics. Despite these strengths, the study is limited in several ways, including lack of generalizability to patients without Medicare as primary insurance source and providers who do not care for Medicare patients, or to providers who care for the veteran population. Our study population included incident dialysis patients who survived the first 6 months of ESRD and were not hospitalized in at least one of months 4 through 6 . We did not have information about provider certification status. We could not determine whether noncomprehensive visits were provided by physician extenders or physician's partners. It is possible that some provider characteristics were misclassified. The calculated distance from provider's office to dialysis unit was based on the provider's billing zip code; many practices have multiple sites, only one of which is used for billing. 
Associations between the type of practice variable and mortality and hospitalization violated the proportionality assumption, and results for the variable should be interpreted with caution. Because our cohort included providers from across the United States, it is possible that our null findings are due to nationwide heterogeneity in practices and outcomes. As we performed multiple analyses, there is potential for spurious associations. Because this is an observational study, despite adjustment for observed confounders, bias due to unobserved confounders likely remains.

In conclusion, no provider characteristics were independently associated with patient mortality. Several characteristics were associated with differences in hospitalization, waitlisting for kidney transplant, and influenza vaccinations. Future research should continue to identify best patient care patterns and implementation strategies aimed at dissemination of best practices across various providers and geographic locations.

\section{Acknowledgements}

The authors thank Chronic Disease Research Group colleagues Delaney Berrini, BS, for manuscript preparation and Nan Booth, MSW, MPH, ELS, for manuscript editing.

\section{Disclosure Statement}

This study was supported by National Institute of Diabetes and Digestive and Kidney Diseases/National Institute of Health R01DK082415. The authors have no conflicts of interest with its subject matter.

\section{References}

1 Reid RO, Friedberg MW, Adams JL, McGlynn EA, Mehrotra A: Associations between physician characteristics and quality of care. Arch Intern Med 2010;170:1442-1449.

- Choudhry NK, Fletcher RH, Soumerai SB: Systematic review: the relationship between clinical experience and quality of health care. Ann Intern Med 2005;142:260-273.

3 Pham HH, Schrag D, Hargraves JL, Bach PB: Delivery of preventive services to older adults by primary care physicians. JAMA 2005;294: 473-481.

4 Ko DT, Austin PC, Chan BT, Tu JV: Quality of care of international and Canadian medical graduates in acute myocardial infarction. Arch Intern Med 2005;165:458-463.

5 Norcini JJ, Kimball HR, Lipner RS: Certification and specialization: do they matter in the outcome of acute myocardial infarction? Acad Med 2000;75:1193-1198.

-6 Chen J, Radford MJ, Wang Y, Krumholz HM: Care and outcomes of elderly patients with acute myocardial infarction by physician specialty: the effects of comorbidity and functional limitations. Am J Med 2000;108:460469.

7 Frances CD, Go AS, Dauterman KW, Deosaransingh K, Jung DL, Gettner S, Newman JM, Massie BM, Browner WS: Outcome following acute myocardial infarction: are differences among physician specialties the result of quality of care or case mix? Arch Intern Med 1999; 159:1429-1436.
8 O’Neill L, Lanska DJ, Hartz A: Surgeon characteristics associated with mortality and morbidity following carotid endarterectomy. Neurology 2000;55:773-781.

$\checkmark 9$ Harley KT, Streja E, Rhee CM, Molnar MZ, Kovesdy CP, Amin AN, Kalantar-Zadeh K: Nephrologist caseload and hemodialysis patient survival in an urban cohort. J Am Soc Nephrol 2013;24:1678-1687.

10 US Renal Data System. Researcher's Guide to the USRDS Database: 2009 ADR Edition. National Institutes of Health, National Institute of Diabetes and Digestive and Kidney Diseases. http://www.usrds.org/2009/fb/index_rg.html (accessed March 14, 2014).

-11 Liu J, Huang Z, Gilbertson DT, Foley RN, Collins AJ: An improved comorbidity index for outcome analyses among dialysis patients. Kidney Int 2010;77:141-151.

12 Ward MM: Socioeconomic status and the incidence of ESRD. Am J Kidney Dis 2008;51: 563-572.

13 US Census Bureau. US Census Bureau Data Set. 2000. http://www.census.gov/main/www/ cen2000.html (accessed March 14, 2014).

14 Hartz AJ, Kuhn EM, Pulido J: Prestige of training programs and experience of bypass surgeons as factors in adjusted patient mortality rates. Med Care 1999;37:93-103.

15 Birkmeyer JD, Stukel TA, Siewers AE, Goodney PP, Wennberg DE, Lucas FL: Surgeon volume and operative mortality in the United States. N Engl J Med 2003;349:2117-2127.
16 Davidson W, Molloy DW, Bedard M: Physician characteristics and prescribing for elderly people in New Brunswick: relation to patient outcomes. CMAJ 1995;152:12271234.

17 Aubin M, Vezina L, Fortin JP, Bernard PM: Effectiveness of a program to improve hypertension screening in primary care. CMAJ 1994;150:509-515.

18 Czaja R, McFall SL, Warnecke RB, Ford L, Kaluzny AD: Preferences of community physicians for cancer screening guidelines. Ann Intern Med 1994;120:602-608.

19 Ayanian JZ, Hauptman PJ, Guadagnoli E, Antman EM, Pashos CL, McNeil BJ: Knowledge and practices of generalist and specialist physicians regarding drug therapy for acute myocardial infarction. N Engl J Med 1994;331: 1136-1142.

20 Streja DA, Rabkin SW: Factors associated with implementation of preventive care measures in patients with diabetes mellitus. Arch Intern Med 1999;159:294-302.

21 Tonelli M, Klarenbach S, Rose C, Wiebe N, Gill J: Access to kidney transplantation among remote- and rural-dwelling patients with kidney failure in the United States. JAMA 2009;301:1681-1690.

22 Maripuri S, Arbogast P, Ikizler TA, Cavanaugh KL: Rural and micropolitan residence and mortality in patients on dialysis. Clin J Am Soc Nephrol 2012;7:1121-1129. 